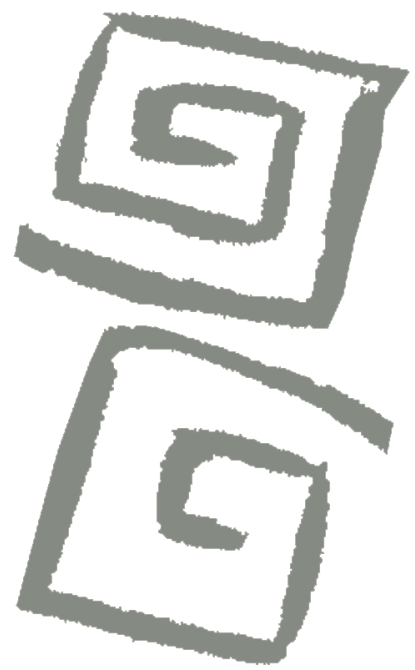

\title{
Dolor cervical entre agricultores que producen tabaco en el sur de Brasil
}

\author{
Neck pain among tobacco farm workers in Southern \\ Brazil
}

Anaclaudia Gastal Fassa', Nadia Spada Fiori², Rodrigo Dalke Meucci , Neice Müller Xavier Faria ${ }^{4}$, Maitê Peres de Carvalho

${ }^{1}$ Autora de correspondencia Doctora en Epidemiología. Profesora Titular, Universidad Federal de Pelotas, Pelotas, Brasil. $\square$ iD

${ }^{2}$ Doctora en Epidemiología. Profesora Adjunta, Universidad Federal de Pelotas, Pelotas, Brasil. $\triangle$ iD

${ }^{3}$ Doctor en Epidemiología. Profesor Adjunto, Universidad Federal do Rio Grande, Rio Grande, Brasil. $\square$ (iD)

${ }^{4}$ Doctora en Epidemiología. Médica Ocupacional, Departamento de Salud del Municipio de Bento Gonçalves, Brasil. $\square$ (iD)

${ }^{5}$ Doctora en Ciencias de la Salud. Investigadora, Universidad Federal de Pelotas, Pelotas, Brasil. $\bowtie$ iD
RESUMEN Este estudio tiene como objetivo evaluar la prevalencia del dolor cervical y los factores asociados entre agricultores que producen tabaco. Se realizó un estudio transversal en el que participaron 2.469 agricultores que producen tabaco en el sur de Brasil. Para la caracterización del dolor cervical se utilizó una adaptación del cuestionario nórdico para síntomas musculoesqueléticos. El análisis multivariante se realizó mediante la regresión de Poisson, siguiendo un modelo teórico jerárquico. La prevalencia del dolor cervical en el año previo entre la población estudiada fue del 7,4\%. Las variables que se asociaron con el dolor cervical entre las mujeres trabajadoras fueron la edad, el consumo de tabaco, el enfardado del tabaco, el uso de motosierras pesadas, trabajar en un ritmo intenso o acelerado y la enfermedad del tabaco verde, mientras que, entre los varones, fueron la edad, el uso de motosierras pesadas, el trabajo sentado en el suelo, la intoxicación por plaguicidas y la enfermedad del tabaco verde. El estudio refuerza la importancia de las cargas de trabajo ergonómicas y fisiológicas en la determinación del dolor cervical. Se necesitan estudios futuros para comprender el papel de la exposición a los plaguicidas y a la nicotina en los problemas musculoesqueléticos. La mecanización de la cosecha del tabaco podría reducir la exposición ergonómica y química, mejorando así la salud de los agricultores.

PALABRAS CLAVES Dolor Cervical; Salud Laboral; Agricultura; Tabaco; Salud Rural; Brasil.

ABSTRACT This study aims to assess neck pain prevalence and associated factors among tobacco farm workers. This is a cross-sectional study of 2,469 tobacco farm workers in southern Brazil. An adapted version of the Nordic Questionnaire of Musculoskeletal Symptoms was used to characterize neck pain. Multivariate analysis was performed using Poisson regression, following a hierarchical theoretical model. Neck pain prevalence in the last year among the population studied was $7.4 \%$. Worker age, tobacco smoking, tobacco bundling, use of heavy chainsaws, working at an intense or accelerated pace and green tobacco sickness were variables associated with neck pain in females. Among males, age, use of heavy chainsaws, working in a sitting position on the ground, pesticide poisoning, and green tobacco sickness were associated with the outcome. The study reinforces the importance of ergonomic and physiological workloads in the determination of neck pain. Future studies are needed to understand the role of pesticides and nicotine exposures on musculoskeletal problems. The mechanization of tobacco harvesting could reduce ergonomic and chemical exposure, thereby improving farmers' health.

KEY WORDS Cervical Pain; Occupational Health; Agriculture; Tobacco; Rural Health; Brazil. 


\section{INTRODUCCIÓN}

Pese a que el sector tabacalero global se enfrenta a una reducción en el consumo y en la producción -debida a una legislación restrictiva y condiciones climáticas desfavorables- China y Brasil cosechan el equivalente a 2.685.983 toneladas de hojas de tabaco por año. El cultivo del tabaco tiene un papel importante en la economía brasileña; el país se destaca como el principal exportador y el segundo productor más grande de tabaco, y el sector genera de forma directa unos 640.000 puestos de trabajo $^{(1)}$. Aproximadamente el $98 \%$ de la cosecha de tabaco en 2017 y 2018 correspondió a la región sur, que produjo el equivalente a 685.983 toneladas de tabaco, y el estado de Rio Grande do Sul lideró la región con el 46,5\% de esta producción ${ }^{(1)}$.

Distintos estudios indican que el dolor cervical es prevalente en el sector agrícola y ganadero y puede restringir la capacidad de trabajar. Se ha observado que afecta el 33\% de ordeñadoras mujeres ${ }^{(2)}$ y el $26 \%$ de trabajadores rurales ${ }^{(3)}$. Los factores ocupacionales asociados con síntomas de dolor en los hombros, el cuello y la espalda inferior incluyen en el trabajo manual, la exposición a las vibraciones, los movimientos repetitivos y las malas condiciones laborales $s^{(4,5,6,7)}$.

En Brasil, el cultivo de tabaco se realiza a través del sistema integrado de producción, una asociación comercial entre productores familiares y firmas que provee asistencia técnica, apoyo financiero y garantías de compra del producto $^{(8)}$. La producción de tabaco se realiza durante todo el año en cinco etapas: la siembra en semillero y preparación del suelo, el trasplante, el cultivo y recolección, el curado y la preclasificación.

La recolección es la etapa que requiere el trabajo más intensivo, con jornadas largas para toda la fuerza de trabajo disponible en la propiedad agrícola. Se trata de un proceso manual que se realiza en distintos pasos, comenzando con las hojas más cercanas al suelo y terminando con las que están en la parte más alta de la planta. Las hojas se Ilevan al galpón, donde trabajadores sentados en el suelo ensartan las hojas, las colocan sobre barras, las cuelgan para el secado y, posteriormente, las enfardan ${ }^{(9)}$. Estos trabajadores realizan una labor extenuante, con posturas incómodas, expuestos a químicos (la nicotina en particular) y a otro tipo de tareas que pueden relacionarse con síntomas musculoesqueléticos ${ }^{(9)}$. A pesar de ello, en la bibliografía no se han reportado síntomas musculoesqueléticos entre agricultores que producen tabaco. Los escasos estudios que se enfocan en esta población abordan la enfermedad del tabaco verde, que resulta de la absorción de nicotina por la piel cuando entra en contacto con las hojas de tabaco.

Este estudio forma parte de un proyecto más grande titulado "La enfermedad del tabaco verde en trabajadores rurales", que evaluó la salud mental, sibilancias, enfermedad del tabaco verde, lumbalgia crónica y otros problemas de salud entre agricultores que producen tabaco. Teniendo en cuenta la importancia económica del cultivo de tabaco en Brasil, el número de trabajadores de la agricultura familiar expuestos, y el hecho de que la cosecha del tabaco involucra trabajo manual intensivo, este trabajo evaluó la prevalencia de dolor cervical y factores asociados entre agricultores que producen tabaco en el sur de Brasil.

\section{MÉTODOS}

Se realizó un estudio transversal con agricultores que producen tabaco en el municipio de São Lourenço do Sul (Rio Grande do Sul, Brasil). Los datos fueron recolectados entre enero y marzo de 2011, durante el período de cosecha.

El estudio se basó en una muestra de 2.469 agricultores que producen tabaco ${ }^{(10)}$ de 18 años o más. A partir de la muestra se estimó una prevalencia de dolor cervical del $7 \%$, con una precisión de \pm 2 puntos porcentuales, un nivel de confianza del $95 \%$ y un poder estadístico del $80 \%$ para identificar razones de prevalencia de alrededor de 2,0 para investigar los factores asociados a 
la mayoría de las asociaciones examinadas, salvo el trabajo en posición inclinada y la intoxicación por plaguicidas en el año previo.

La muestra se diseñó a partir de 3.852 facturas de venta de tabaco emitidas en 2009, proporcionadas al equipo de investigación por el Departamento de Finanzas del Municipio de São Lourenço do Sul. Considerando el tamaño deseado de la muestra y la estimación de aproximadamente tres trabajadores por propiedad agrícola, se seleccionaron 1.100 facturas de forma aleatoria. Con el apoyo de informantes claves, se procedió a identificar la ubicación de las propiedades.

Siguiendo los criterios establecidos por el Instituto Brasileño de Geografía y Estadística para considerar a un individuo como un trabajador rural en sus encuestas, en este estudio se incluyeron los individuos que trabajaban en la producción de tabaco al menos 15 horas por semana al momento de ser encuestados $^{(12,13)}$. En los casos en que el individuo había sido trabajador de la producción de tabaco en 2009, pero ya no se encontraba realizando esa actividad en el momento de la encuesta, se reemplazó por el vecino más cercano que cultivase tabaco. Se consideró que una propiedad era inelegible para la inclusión en el estudio si el individuo que emitía la factura ya no se dedicaba a la producción de tabaco o en cuya propiedad no se encontraba nadie que se dedicara a la producción de tabaco, o el individuo residía en un área urbana del municipio o se había mudado a otro municipio. En casos en que no se pudo constatar la ubicación de una propiedad agrícola de tabaco, se consideró una pérdida de 2,7 personas por propiedad. Este número fue sumado al número de individuos que no se encontraban en las propiedades o que optaron por no participar, para evaluar la tasa de respuesta.

Para la recolección de datos se utilizaron dos instrumentos: uno para los agricultores individuales y otro para las propiedades de agricultura familiar. Ambos fueron precodificados en formato electrónico para ser usados con asistentes digitales personales (PDA). El instrumento utilizado para las propiedades indagó en factores socioeconómicos y fue respondido por el principal administrador de la propiedad, miembro de la familia de agricultores. El instrumento aplicado a agricultores individuales abordó factores socioeconómicos y demográficos (edad, nivel educativo alcanzado, tiempo que trabaja con el tabaco, producción de tabaco); aspectos comportamentales (uso de tabaco, abuso del alcohol); tareas laborales (uso de motosierras pesadas, enfardado y transporte de hojas de tabaco, preparación del terreno para sembrar el tabaco, trabajo en el galpón de curado, aplicación de plaguicidas y/o herbicidas, ensartado de las hojas de tabaco, cosecha de hojas cercanas al suelo, subir a la parte alta del galpón, alzar y colgar las barras con las hojas ensartadas); cargas de trabajo (trabajar en un ritmo intenso o acelerado, cantidad de horas trabajadas durante la cosecha, peso promedio que se carga durante el trabajo, trabajo extenuante, trabajo en una posición inclinada, trabajo sentado en el suelo), comorbilidades (enfermedad del tabaco verde, intoxicación por plaguicidas); y dolor en la columna vertebral.

Se consideró fumador a aquella persona que fumó uno o más cigarrillos al menos un día en el mes previo a ser entrevistada y exfumador a aquella persona que reportó haber dejado de fumar más de un mes antes de ser entrevistada. A los fines del presente análisis, la variable tabaquismo fue tratado como una variable dicotómica (no fumadores y fumadores/ exfumadores). El trabajo intenso, extenuante o en un ritmo acelerado fue auto-calificado. Se evaluó la intoxicación por plaguicidas a través de la pregunta " ¿Tuvo intoxicación por plaguicidas alguna vez en su vida?"(14). La enfermedad del tabaco verde (GTS, por sus siglas en inglés) se definió como la ocurrencia de mareos o dolores de cabeza y náuseas o vómitos dentro de dos días después de cosechar tabaco a lo largo del año previo ${ }^{(15)}$.

Se utilizó una versión adaptada del "Cuestionario nórdico para síntomas musculoesqueléticos"(16) para caracterizar el dolor cervical. Este cuestionario ha sido validado en Brasil(17) y utilizado en otros estudios en el país ${ }^{(18,19)}$. Para evaluar el dolor cervical, se les preguntó a los agricultores que producen 
tabaco si habían tenido dolor de espalda en los 12 meses previos a la entrevista. A los que reportaron haber tenido dolor, se les mostró una figura humana en posición vertical con las regiones cervical, dorsal y lumbar pintados de distintos colores y se consideró que los que señalaron la región cervical habían tenido dolor cervical.

Los análisis estadísticos se realizaron con Stata $12.0^{\circ}$. En primer lugar, se verificó la distribución de frecuencias de cada variable, examinando medidas de tendencia central y proporciones, así como la prueba de chi cuadrado de heterogeneidad para evaluar la diferencia de cada variable entre los sexos. Luego, se llevó a cabo el análisis bivariado, probando la asociación entre las variables independientes y la variable dependiente, y usando la prueba de Wald para heterogeneidad y tendencia lineal. El análisis multivariante se realizó mediante regresión de Poisson con varianza robusta y selección hacia atrás, para estimar las razones de prevalencia (RP) e intervalos de confianza (IC 95\%). Se incluyeron en el modelo las variables con un valor de $p$ igual o menor a 0,20 a fin de controlar factores de confusión. Se consideró que una asociación fue significativa cuando el valor de $p$ era igual o menor a 0,05. El análisis multivariante siguió un modelo jerárquico ${ }^{(20)}$ con cinco niveles: 1) factores socioeconómicos y demográficos; 2) aspectos comportamentales; 3) tareas laborales; 4) cargas de trabajo; y 5) comorbilidades (Figura 1).

Este estudio fue aprobado por el Comité de Ética en Investigación de la Universidad Federal de Pelotas (Informe No. 11/2010) y todos los participantes firmaron los términos del consentimiento libre e informado.

\section{RESULTADOS}

La muestra quedó conformada por 2.469 individuos que trabajaban en 912 propiedades. La tasa de respuesta fue del $94,1 \%$. De la población bajo estudio, el 59,3\% eran varones y la mitad tenía entre 18 y 39 años de edad; el $4,8 \%$ eran mujeres, y el $7,2 \%$ de los

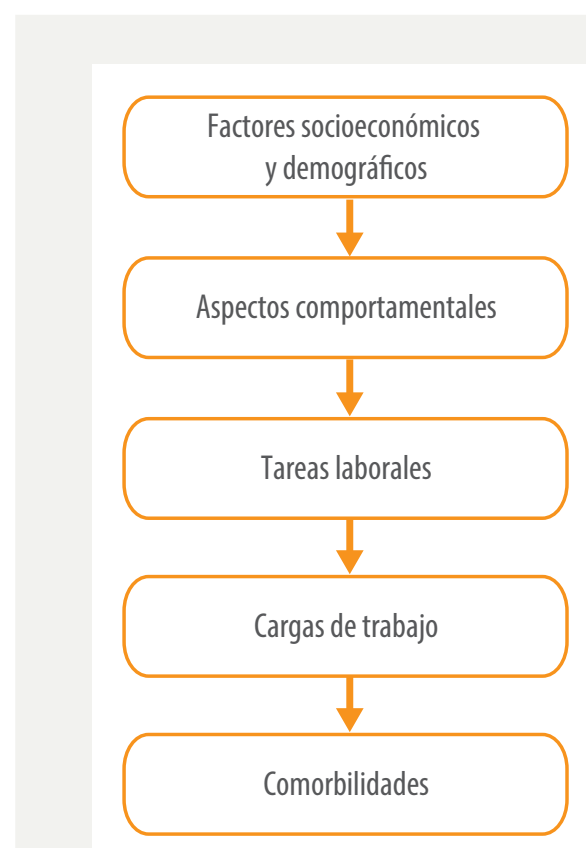

Figura 1. Modelo jerárquico para el análisis multivariante.

Fuente: Elaboración propia.

varones tenían más de 60 años. La proporción de individuos con hasta 4 años de escolaridad $(44 \%)$ fue similar en ambos sexos, más del $60 \%$ había trabajado en la producción de tabaco durante al menos 10 años, y el $44 \%$ trabajaba en propiedades que producían entre $5.001 \mathrm{~kg}$ y $10.000 \mathrm{~kg}$ de tabaco por año (Tabla 1).

Entre las mujeres, el 7,6\% eran fumadoras o exfumadoras, mientras que la mitad de los varones tenía esta condición. El enfardado de las hojas de tabaco fue una tarea laboral realizada por un número importante de trabajadores de ambos sexos $(70,6 \%$ de mujeres y $87,2 \%$ de varones). Colocar las hojas de tabaco en barras para colgar en los galpones de curado fue una tarea que predominó entre las mujeres $(73,1 \%)$; mientras que el uso de motosierras pesadas $(45,4 \%)$ y la preparación del suelo para la siembra de tabaco $(83,3 \%)$ fueron tareas que predominaban entre los varones. Más de la mitad de los agricultores que producían tabaco de ambos sexos trabajaban entre 9 y 12 horas por día durante la cosecha, y más de la mitad reportó 
Tabla 1. Número y distribución porcentual (intervalos de confianza del 95\%) de los factores socioeconómicos y demográficos, aspectos comportamentales, tareas laborales, cargas de trabajo, comorbilidades y dolor cervical según sexo en agricultores que producen tabaco. Municipio de São Lourenço do Sul, Rio Grande do Sul, Brasil, 2011. ( $n=2.469)$.

\begin{tabular}{|c|c|c|c|c|c|c|c|}
\hline \multirow{2}{*}{ Variables } & \multicolumn{3}{|c|}{ Mujeres } & \multicolumn{3}{|c|}{ Varones } & \multirow[t]{2}{*}{ Valor de $p^{*}$} \\
\hline & $\mathrm{n}$ & $\%$ & IC $95 \%$ & $\mathrm{n}$ & $\%$ & IC $95 \%$ & \\
\hline \multicolumn{8}{|c|}{ Factores socioeconómicos y demográficos } \\
\hline \multicolumn{8}{|l|}{ Edad } \\
\hline 18-39 años & 522 & 51,9 & $48,8-55,0$ & 746 & 51,0 & $48,4-53,5$ & \multirow{3}{*}{0,045} \\
\hline 40-59 años & 435 & 43,3 & $40,2-46,3$ & 612 & 41,8 & $39,3-44,3$ & \\
\hline 60 años o más & 48 & 4,8 & $3,4-6,1$ & 106 & 7,2 & $5,9-8,6$ & \\
\hline \multicolumn{8}{|l|}{ Nivel educativo } \\
\hline $0-4$ años & 442 & 44,0 & $41,0-47,0$ & 644 & 44,0 & $41,4-46,5$ & \multirow{3}{*}{0,016} \\
\hline 5-8 años & 473 & 47,1 & $44,0-50,1$ & 732 & 50,0 & $47,4-52,6$ & \\
\hline 9 años o más & 90 & 9,0 & $7,2-10,7$ & 88 & 6,0 & $4,8-7,2$ & \\
\hline \multicolumn{8}{|l|}{ Tiempo trabajando con el tabaco } \\
\hline Hasta 9 años & 311 & 31,0 & $28,1-33,9$ & 457 & 31,2 & $28,9-33,6$ & \multirow{3}{*}{0,136} \\
\hline 10-19 años & 347 & 34,6 & $31,6-37,5$ & 455 & 31,1 & $28,7-33,5$ & \\
\hline 20 años o más & 345 & 34,4 & $31,4-37,3$ & 551 & 37,7 & $35,2-40,1$ & \\
\hline \multicolumn{8}{|l|}{ Producción de tabaco } \\
\hline $1-5.000 \mathrm{~kg}$ & 359 & 36,0 & $33,0-38,9$ & 487 & 33,5 & $31,0-35,9$ & \\
\hline $5.001-10.000 \mathrm{~kg}$ & 438 & 44,0 & $33,0-38,9$ & 638 & 43,8 & $41,3-46,4$ & 0,242 \\
\hline $10.001-36.000 \mathrm{~kg}$ & 201 & 20,1 & $17,6-22,6$ & 330 & 22,7 & $20,5-24,8$ & \\
\hline Aspectos comportamentale & & & & & & & \\
\hline Consumo de tabaco & & & & & & & \\
\hline No fuma & 929 & 92,4 & $90,8-94,1$ & 730 & 49,9 & $47,3-52,4$ & \\
\hline Ex-fumador/a & 44 & 4,4 & $3,1-5,6$ & 278 & 19,0 & $17,0-21,0$ & $<0,001$ \\
\hline Fumador/a & 32 & 3,2 & $2,1-4,3$ & 456 & 31,1 & $28,8-33,5$ & \\
\hline Tareas laborales & & & & & & & \\
\hline Enfardado de hojas de tabaco & & & & & & & \\
\hline No / A veces & 295 & 29,4 & $26,6-32,3$ & 187 & 12,8 & $11,1-14,5$ & -0001 \\
\hline Con frecuencia / Siempre & 707 & 70,6 & $67,7-73,4$ & 1.275 & 87,2 & $85,5-89,0$ & $<0,001$ \\
\hline Preparación del suelo para sem & & & & & & & \\
\hline No / A veces & 464 & 46,2 & $43,1-49,2$ & 244 & 16,7 & $14,8-18,6$ & \\
\hline Con frecuencia / Siempre & 541 & 53,8 & $50,7-56,9$ & 1.219 & 83,3 & $81,4-85,2$ & $<0,001$ \\
\hline Uso de motosierras pesadas & & & & & & & \\
\hline No & 923 & 95,6 & $94,3-96,9$ & 793 & 54,6 & $52,0-57,1$ & $<0001$ \\
\hline Sí & 42 & 4,3 & $3,1-5,6$ & 660 & 45,4 & $42,9-48,0$ & $<0,001$ \\
\hline Colocar hojas de tabaco en barı & & & & & & & \\
\hline № & 270 & 26,9 & $24,1-29,6$ & 943 & 64,5 & $62,0-66,9$ & $<0001$ \\
\hline Sí & 735 & 73,1 & $70,4-75,9$ & 520 & 35,5 & $33,1-38,0$ & $<0,001$ \\
\hline Horas trabajadas durante la cos & & & & & & & \\
\hline Hasta 8 horas & 195 & 19,4 & $17,0-21,9$ & 124 & 8,5 & $7,1-9,9$ & \\
\hline $9-12$ horas & 556 & 55,4 & $52,3-58,5$ & 805 & 55,2 & $52,6-57,7$ & $<0,001$ \\
\hline 13-18 horas & 252 & 25,1 & $22,4-27,8$ & 530 & 36,3 & $33,8-38,8$ & \\
\hline Cargas de trabajo & & & & & & & \\
\hline Trabajo en un ritmo intenso $0 \mathrm{a}$ & & & & & & & \\
\hline Hasta 3 meses ritmo pesado & 434 & 43,3 & $40,2-46,4$ & 600 & 41,1 & $38,6-43,7$ & \\
\hline 4-7 meses ritmo pesado & 469 & 46,8 & $43,7-49,9$ & 687 & 47,1 & $44,5-49,7$ & 0,284 \\
\hline 8 meses o más & 99 & 9,9 & $8,0-11,7$ & 171 & 11,7 & $10,1-13,4$ & \\
\hline Trabajo en una posición inclina & & & & & & & \\
\hline No & 78 & 7,8 & $6,1-9,4$ & 94 & 6,4 & $5,2-7,7$ & 194 \\
\hline Sí & 925 & 92,2 & $90,6-93,9$ & 1.370 & 93,6 & $92,3-94,8$ & 0,194 \\
\hline Trabajo extenuante & & & & & & & \\
\hline No & 520 & 51,8 & $48,7-54,9$ & 581 & 39,7 & $37,2-42,2$ & $<0001$ \\
\hline Sí & 484 & 48,2 & $45,1-51,3$ & 883 & 60,3 & $57,8-62,8$ & $<0,001$ \\
\hline Trabajo sentado en el suelo & & & & & & & \\
\hline No & 486 & 48,4 & $45,3-51,5$ & 770 & 52,6 & $50,0-55,1$ & 001 \\
\hline Sí & 518 & 51,6 & $48,5-54,7$ & 694 & 47,4 & $44,8-50,0$ & 0,041 \\
\hline Comorbilidades & & & & & & & \\
\hline Intoxicación por plaguicidas en & & & & & & & \\
\hline No & 1.001 & 99,7 & $99,4-100,0$ & 1.456 & 99,4 & $99,1-99,8$ & 0364 \\
\hline Sí & 3 & 0,3 & $0,0-0,6$ & 8 & 0,5 & $0,2-0,9$ & 0,304 \\
\hline Enfermedad del tabaco verde e & & & & & & & \\
\hline Nunca & 846 & 85,0 & $82,8-87,2$ & 1.324 & 91,2 & $89,7-92,6$ & \\
\hline Hasta 3 episodios & 73 & 7,3 & $5,7-8,9$ & 88 & 6,1 & $4,8-7,3$ & $<0,001$ \\
\hline 40 más episodios & 76 & 7,6 & $6,0-9,3$ & 40 & 2,7 & $1,9-3,6$ & \\
\hline Variable dependiente & & & & & & & \\
\hline Dolor cervical & & & & & & & \\
\hline No & 913 & 90,9 & $89,1-92,7$ & 1.372 & 93,8 & $92,5-95,0$ & 0,008 \\
\hline Sí & 91 & 9,1 & $7,3-10,8$ & 91 & 6,2 & $5,0-7,4$ & 0,008 \\
\hline
\end{tabular}

Fuente: Elaboración propia.

Nota: Las variables independientes están estructuradas en un modelo jerárquico de cinco niveles: 1) factores socioeconómicos y demográficos; 2) aspectos comportamentales; 3) tareas laborales; 4) cargas de trabajo; 5) comorbilidades.

IC $95 \%=$ Intervalo de confianza del $95 \%$.

*Prueba de chi cuadrado para heterogeneidad. 
trabajar en un ritmo intenso o acelerado durante cuatro meses al año o más. Entre los varones, el $60 \%$ realizó trabajo extenuante, en comparación con el $48 \%$ de las mujeres. Más del 92\% de ambos sexos trabajó en una posición inclinada, y el 50\% trabajó sentado en el suelo. En relación con las comorbilidades, durante el año previo a la entrevista, la prevalencia de intoxicación por plaguicidas fue del $0,3 \%$ en mujeres y $0,5 \%$ en varones, y la prevalencia de 4 o más episodios de la enfermedad del tabaco verde fue del 7,6\% en mujeres y $2,7 \%$ en varones (Tabla 1 ). La prevalencia de dolor cervical en el año previo en la población estudiada fue del $7,4 \%$, con una diferencia significativa entre los sexos $(p=0,008)$ : el $9,1 \%$ en mujeres y el $6,2 \%$ en varones.

En mujeres, el análisis ajustado por el conjunto de variables incluidas en el modelo (Tabla 2) mostró que la razón de prevalencia de dolor cervical fue de 1,7 más para mujeres entre 40 y 59 años de edad con relación a las mujeres más jóvenes, y aproximada-

Tabla 2. Razones de prevalencia crudas y ajustadas (intervalos de confianza del 95\%) a partir de la regresión de Poisson, cuyo modelo incluye factores socioeconómicos y demográficos, aspectos comportamentales, tareas laborales, cargas de trabajo, comorbilidades y dolor cervical en mujeres agricultoras que producen tabaco. Municipio de São Lourenço do Sul, Rio Grande do Sul, Brasil, 2011. ( $n=1.005)$.

\begin{tabular}{|c|c|c|c|c|c|c|c|}
\hline \multirow{2}{*}{ Variables } & \multirow{2}{*}{$\%$} & \multicolumn{3}{|c|}{ Razón de prevalencia cruda } & \multicolumn{3}{|c|}{ Razón de prevalencia ajustada } \\
\hline & & $\mathrm{RP}$ & $\mathrm{IC} 95 \%$ & Valor de $p$ & $\mathrm{RP}$ & IC $95 \%$ & Valor de $p$ \\
\hline \multicolumn{8}{|c|}{ Factores socioeconómicos y demográficos } \\
\hline \multicolumn{8}{|l|}{ Edad } \\
\hline $18-39$ años ${ }^{1}$ & 6,9 & 1,00 & - & \multirow{3}{*}{$0,023^{*}$} & 1,00 & - & \multirow{3}{*}{$0,023^{*}$} \\
\hline 40-59 años & 11,9 & 1,73 & $1,15-2,60$ & & 1,73 & $1,15-2,60$ & \\
\hline 60 años o más & 6,4 & 0,92 & $0,30-2,89$ & & 0,92 & $0,30-2,89$ & \\
\hline \multicolumn{8}{|l|}{ Aspectos comportamentales } \\
\hline \multicolumn{8}{|l|}{ Consumo de tabaco } \\
\hline No fuma ${ }^{1}$ & 8,4 & 1,00 & - & \multirow{2}{*}{$0,010^{*}$} & 1,00 & - & \multirow{2}{*}{$0,011^{*}$} \\
\hline Fumadora / Ex-fumadora & 17,1 & 2,03 & $1,19-3,49$ & & 2,00 & $1,17-3,41$ & \\
\hline \multicolumn{8}{|l|}{ Tareas laborales } \\
\hline \multicolumn{8}{|l|}{ Enfardado de hojas de tabaco } \\
\hline No / $A$ veces ${ }^{1}$ & 5,1 & 1,00 & - & \multirow{2}{*}{$0,006^{*}$} & 1,00 & - & \multirow{2}{*}{$0,010^{*}$} \\
\hline Con frecuencia / Siempre & 10,8 & 2,12 & $1,24-3,62$ & & 2,05 & $1,19-3,52$ & \\
\hline \multicolumn{8}{|l|}{ Uso de motosierras pesadas } \\
\hline $\mathrm{No}^{1}$ & 8,5 & 1,00 & - & \multirow{2}{*}{$0,003^{*}$} & 1,00 & - & \multirow{2}{*}{$0,027^{*}$} \\
\hline Sí & 21,4 & 2,53 & $1,37-4,69$ & & 2,05 & $1,08-3,86$ & \\
\hline \multicolumn{8}{|l|}{ Cargas de trabajo } \\
\hline \multicolumn{8}{|c|}{ Trabajo en un ritmo intenso o acelerado } \\
\hline Hasta 3 meses ritmo pesado ${ }^{1}$ & 6,0 & 1,00 & - & \multirow{3}{*}{$0,001^{* *}$} & 1,00 & - & \multirow{3}{*}{$0,033^{* *}$} \\
\hline 4-7 meses ritmo pesado & 10,7 & 1,77 & $1,12-2,80$ & & 1,54 & $0,97-2,42$ & \\
\hline 8 meses o más & 15,1 & 2,52 & $1,39-4,58$ & & 1,77 & $0,93-3,38$ & \\
\hline \multicolumn{8}{|l|}{ Trabajo en una posición inclinada } \\
\hline $\mathrm{No}^{1}$ & 2,6 & 1,00 & - & \multirow{2}{*}{$0,061^{*}$} & 1,00 & - & \multirow{2}{*}{$0,109^{*}$} \\
\hline Sí & 9,6 & 3,75 & $0,94-14,96$ & & 3,27 & $0,77-13,91$ & \\
\hline \multicolumn{8}{|l|}{ Comorbilidades } \\
\hline \multicolumn{8}{|c|}{ Enfermedad del tabaco verde en el año previo } \\
\hline Nunca $^{1}$ & 7,9 & 1,00 & - & \multirow{3}{*}{$0,002^{* *}$} & 1,00 & - & \multirow{3}{*}{$0,005^{* *}$} \\
\hline Hasta 3 episodios & 11,0 & 1,38 & $0,69-2,77$ & & 1,41 & $0,70-2,83$ & \\
\hline 40 más episodios & 18,4 & 2,33 & $1,37-3,94$ & & 2,15 & $1,25-3,70$ & \\
\hline
\end{tabular}

Fuente: Elaboración propia.

Nota: Las variables independientes están estructuradas en un modelo jerárquico de cinco niveles: 1) factores socioeconómicos y demográficos; 2) aspectos comportamentales; 3) tareas laborales; 4) cargas de trabajo; 5) comorbilidades.

IC 95\%= Intervalo de confianza del 95\%. RP = Razón de prevalencia.

Valor de referencia.

* Prueba de Wald para heterogeneidad.

**Prueba de Wald para tendencia lineal. 
Tabla 3. Razones de prevalencia crudas y ajustadas (intervalos de confianza del 95\%) a partir de la regresión de Poisson, cuyo modelo incluye factores socioeconómicos y demográficos, aspectos comportamentales, tareas laborales, cargas de trabajo, comorbilidades y dolor cervical en varones agricultores que producen tabaco. Municipio de São Lourenço do Sul, Rio Grande do Sul, Brasil, 2011. ( $n=1.464)$.

\begin{tabular}{|c|c|c|c|c|c|c|c|}
\hline \multirow[t]{2}{*}{ Variables } & \multirow[t]{2}{*}{$\%$} & \multicolumn{3}{|c|}{ Razón de prevalencia cruda } & \multicolumn{3}{|c|}{ Razón de prevalencia ajustada } \\
\hline & & RP & IC $95 \%$ & Valor de $p$ & RP & IC $95 \%$ & Valor de $p$ \\
\hline \multicolumn{8}{|c|}{ Factores socioeconómicos y demográficos } \\
\hline \multicolumn{8}{|l|}{ Edad } \\
\hline 18-39 años ${ }^{1}$ & 4,4 & 1,00 & - & \multirow{3}{*}{$<0,001^{* *}$} & 1,00 & - & \multirow{3}{*}{$<0,001^{* *}$} \\
\hline 40-59 años & 7,0 & 1,59 & $1,02-2,47$ & & 1,59 & $1,02-2,47$ & \\
\hline 60 años o más & 14,1 & 3,20 & $1,80-5,69$ & & 3,20 & $1,80-5,69$ & \\
\hline \multicolumn{8}{|l|}{ Aspectos comportamentales } \\
\hline \multicolumn{8}{|l|}{ Consumo de tabaco } \\
\hline No fuma ${ }^{1}$ & 4,5 & 1,00 & - & \multirow{2}{*}{$0,008^{*}$} & 1,00 & - & \multirow{2}{*}{$0,063^{*}$} \\
\hline Fumador / Ex-fumador & 7,9 & 1,75 & $1,15-2,65$ & & 1,49 & $0,98-2,28$ & \\
\hline \multicolumn{8}{|l|}{ Tareas laborales } \\
\hline \multicolumn{8}{|c|}{ Preparación del suelo para sembrar tabaco } \\
\hline No / $A$ veces ${ }^{1}$ & 9,4 & 1,00 & - & \multirow{2}{*}{$0,023^{*}$} & 1,00 & - & \multirow{2}{*}{$0,062^{*}$} \\
\hline Con frecuencia / Siempre & 5,6 & 0,59 & $0,38-0,93$ & & 0,63 & $0,39-1,02$ & \\
\hline \multicolumn{8}{|l|}{ Uso de motosierras pesadas } \\
\hline No ${ }^{1}$ & 5,2 & 1,00 & - & \multirow{2}{*}{$0,079^{*}$} & 1,00 & - & \multirow{2}{*}{$0,009^{*}$} \\
\hline Sí & 7,4 & 1,43 & $0,96-2,14$ & & 1,68 & $1,13-2,49$ & \\
\hline \multicolumn{8}{|l|}{ Colocar hojas de tabaco en barras } \\
\hline $\mathrm{No}^{1}$ & 5,3 & 1,00 & - & \multirow{2}{*}{$0,050^{*}$} & 1,00 & - & \multirow{2}{*}{$0,183^{*}$} \\
\hline Sí & 7,9 & 1,49 & $1,00-2,22$ & & 1,31 & $0,88-1,95$ & \\
\hline \multicolumn{8}{|l|}{ Cargas de trabajo } \\
\hline \multicolumn{8}{|c|}{ Trabajo en un ritmo intenso 0 acelerado } \\
\hline Hasta 3 meses ritmo pesado ${ }^{1}$ & 4,7 & 1,00 & - & \multirow{3}{*}{$0,031^{* *}$} & 1,00 & - & \\
\hline 4-7 meses ritmo pesado & 7,1 & 1,53 & $0,97-2,40$ & & 1,49 & $0,93-2,37$ & $0,085^{* *}$ \\
\hline 8 meses o más & 8,2 & 1,75 & $0,94-3,26$ & & 1,56 & $0,83-2,94$ & \\
\hline Trabajo sentado en el suelo & & & & & & & \\
\hline $\mathrm{No}^{1}$ & 7,3 & 1,00 & - & ก $801 *$ & 1,00 & - & ค००47* \\
\hline Sí & 5,0 & 0,69 & $0,46-1,05$ & $0,0811^{*}$ & 0,66 & $0,44-0,99$ & $0,04 \pi^{*}$ \\
\hline Trabajo extenuante & & & & & & & \\
\hline $\mathrm{No}^{\mathrm{P}}$ & 5,0 & 1,00 & - & & 1,00 & - & \\
\hline Sí & 7,0 & 1,40 & $0,91-2,15$ & $0,120^{*}$ & 1,50 & $0,97-2,32$ & $0,067^{*}$ \\
\hline Comorbilidades & & & & & & & \\
\hline Intoxicación por plaguicidas en e & & & & & & & \\
\hline $\mathrm{No}^{1}$ & 6,1 & 1,00 & - & & 1,00 & - & \\
\hline Sí & 25,0 & 4,09 & $1,21-13,81$ & $0,023^{*}$ & 3,85 & $1,05-14,14$ & $0,042^{*}$ \\
\hline Enfermedad del tabaco verde en & & & & & & & \\
\hline Nunca $^{1}$ & 5,6 & 1,00 & - & & 1,00 & - & \\
\hline Hasta 3 episodios & 11,4 & 2,03 & $1,09-3,79$ & $0,001^{* *}$ & 2,07 & $1,08-3,95$ & $0,005^{* *}$ \\
\hline 40 más episodios & 15,0 & 2,68 & $1,24-5,79$ & & 2,21 & $1,05-4,65$ & \\
\hline
\end{tabular}

Fuente: Elaboración propia.

Nota: Las variables independientes están estructuradas en un modelo jerárquico de cinco niveles: 1) factores socioeconómicos y demográficos; 2) aspectos comportamentales; 3) tareas laborales; 4) cargas de trabajo; 5) comorbilidades.

IC 95\%= Intervalo de confianza del 95\%. RP = Razón de prevalencia.

'Valor de referencia.

* Prueba de Wald para heterogeneidad.

**Prueba de Wald para tendencia lineal.

mente el doble para fumadoras/exfumadoras $(R P=2,00)$ y para las mujeres que enfardaban las hojas de tabaco $(\mathrm{RP}=2,05)$ o usaban motosierras pesadas $(\mathrm{RP}=2,05)$. La cantidad de tiempo de trabajo a ritmo intenso o acelerado y el número de episodios de la enfermedad del tabaco verde mostraron asociación positiva con el dolor cervical (Tabla 2).

En varones, el análisis ajustado por el conjunto de variables incluidas en el modelo (Tabla 3) mostró que trabajar sentado en el suelo fue un factor de protección ( $R P=0,66)$, 
mientras que el uso de motosierras pesadas aumentó 1,7 la razón de prevalencia de dolor cervical y haber sufrido intoxicación por plaguicidas durante el año previo acrecentó 3,8 veces la razón de prevalencia de reportar dolor cervical. La edad y el número de episodios de la enfermedad del tabaco verde mostraron asociación lineal positiva con la variable dependiente (Tabla 3).

\section{DISCUSIÓN}

El presente estudio encontró una prevalencia de dolor cervical durante el año previo significativamente más alta entre las mujeres en comparación con los varones. El uso de motosierras pesadas y haber tenido episodios de la enfermedad del tabaco verde durante el año previo fueron variables que mostraron asociación positiva con el dolor cervical en ambos sexos. El hábito de fumar, el enfardado de hojas de tabaco y trabajar a un ritmo intenso o acelerado fueron factores asociados positivamente en las mujeres, mientras que la edad mayor y haber sufrido intoxicación por plaguicidas durante el año previo mostraron asociación positiva con el dolor cervical en los varones.

En estudios sobre agricultores en Corea del Sur y EEUU, la prevalencia de dolor cervical en el año previo varió considerablemente $-21,8 \%$ y $8,9 \%$ respectivamente ${ }^{(21,22)}$ - mientras que nuestro estudio ha encontrado una prevalencia del 7,4\%; sin embargo, estos estudios evaluaron otros cultivos, lo cual dificulta la comparación.

El cultivo de tabaco es principalmente manual, intensivo en trabajo y en gran medida se realiza a través de la producción familiar, con una marcada división sexual del trabajo $^{(23)}$. La construcción de género tiene una dimensión histórica, marcada por factores culturales de larga data respecto a la masculinidad y la ruralidad, y exacerbada por el trabajo físico extenuante necesario en algunas tareas de la producción agrícola ${ }^{(24,25)}$. Algunos estudios epidemiológicos ${ }^{(26,27)}$ indican que factores fisiológicos, hormonales o psicosociales -o hasta factores relacionados con los distintos tipos de tareas realizadas por varones y mujeres- pueden relacionarse con un mayor dolor cervical. Asimismo, las mujeres en general recuerdan más detalles sobre sus problemas de salud que los varones y recurren a la asistencia médica con mayor frecuencia $^{(28)}$. Nuestros hallazgos coinciden con estudios realizados con trabajadores rurales en Suecia y EEUU ${ }^{(2,22)}$ que también reportaron mayor prevalencia de dolor cervical en el año previo entre las mujeres.

La asociación positiva entre edad y dolor cervical es consistente con un estudio que evaluó el dolor musculoesquelético crónico entre trabajadores agrícolas latinoamericanos de ambos sexos y hasta 55 años de edad ${ }^{(22)}$. Es sabido que la degeneración de la columna vertebral ocurre a medida que se gastan los discos intervertebrales, lo cual se exacerba en presencia de osteoporosis, artrosis y osteofitosis, y puede resultar en diversas morbilidades. Hubo un reducido número de trabajadoras mujeres mayores de 60 años de edad. Si bien debería corroborarse con otras investigaciones, esto podría deberse al reemplazo del trabajo agrícola por tareas domésticas, principalmente, si han presentado problemas de salud. Como tal, el efecto del trabajador saludable puede justificar la ausencia de una asociación lineal entre la edad y el dolor cervical entre las mujeres, dado que las mujeres de 40 a 59 años muestran mayor razón de prevalencia. Entre los varones, la tendencia lineal entre la edad y la variable dependiente sugiere que ellos continúan trabajando como agricultores que producen tabaco, a pesar de la presencia de morbilidades.

Este estudio muestra que el enfardado de las hojas de tabaco, aunque era realizado tanto por varones como por mujeres, solo se asoció con el dolor cervical en el caso de las mujeres. Esta tarea requiere esfuerzo físico y movimientos repetitivos al enfardar las hojas de tabaco, lo cual puede afectar más a las mujeres que a los varones, si el peso que deben cargar es mayor a la capacidad que soportan las mujeres, que tienen una estructura fisiológica menos preparada para mayores esfuerzos. Otros estudios también señalan 
asociaciones positivas entre el esfuerzo físico, los movimientos repetitivos y el dolor cervical en las mujeres ${ }^{(6,29)}$.

Trabajar sentado en el suelo fue un factor de protección para el dolor cervical en los varones. Esto puede estar relacionado con el efecto del trabajador saludable, dado que el dolor cervical en los varones puede ser un factor limitante en cuanto a tareas que requieren esta posición.

La asociación positiva entre el uso de motosierras pesadas y el dolor cervical en ambos sexos puede estar relacionada con el peso y las vibraciones intensas de estos equipos. Se reconoce que estos factores son dañinos para las articulaciones y la musculatura ${ }^{(30)}$, dado que provocan contracciones musculares repetitivas $y$, consecuentemente, la fatiga de tejidos, la compresión de nervios y tendones y, en muchos casos, dolor de cabeza. Distintos estudios revelan que las condiciones de trabajo físico que involucran el esfuerzo físico extenuante es un factor de riesgo relevante para síntomas musculoesqueléticos en la región cervical y escapular ${ }^{(31,32)}$.

La asociación lineal positiva entre el tiempo de trabajo a un ritmo intenso o acelerado y el dolor cervical en las mujeres es consistente con hallazgos previamente reportados en la bibliografía. Trabajar muchas horas por períodos largos ${ }^{(33)}$, sobre todo con exposición a tareas físicas como movimientos repetitivos, esfuerzo físico vigoroso, postura inadecuada, vibraciones, o hasta una combinación de estas y otras formas de exposición ${ }^{(29,34)}$ provoca fatiga muscular ${ }^{(35)}$, mental y psicomotora ${ }^{(36)}$. La persistencia de estas exposiciones puede producir fatiga crónica, que puede estar asociada a la fibromialgia(37).

Los agricultores que producen tabaco están sujetos a distintas formas de exposición a la nicotina, ya sea por la piel durante la cosecha o a través de la inhalación de la nicotina del ambiente dentro de los galpones en los que se almacena el tabaco o en áreas cercanas a las plantaciones donde se cultiva( ${ }^{(38)}$. Esta exposición a la nicotina ocupacional se combina con el tabaquismo activo o pasivo, que ocasiona una exposición desmedida a la nicotina. En términos biológicos, es sabido que la presencia de la nicotina en las vías sanguíneas reduce la cantidad de citoquinas proinflamatorias en la circulación, así como la nutrición de los discos intervertebrales, resultados que pueden promover la degeneración de los discos ${ }^{(39)}$. El consumo de tabaco solo se asoció con el dolor cervical en las mujeres, sugiriendo que la susceptibilidad a la nicotina puede diferir entre los sexos. Por otro lado, el número de episodios de la enfermedad del tabaco verde durante el año previo mostró un incremento lineal de la asociación positiva con el dolor cervical en ambos sexos, sugiriendo que el daño causado al organismo por la intoxicación por nicotina puede también ser perjudicial al sistema musculoesquelético.

La intoxicación por plaguicidas durante el año previo mostró una asociación positiva con el dolor cervical en los varones. Los agricultores que producen tabaco, en particular los varones adultos jóvenes, están en contacto directo con los plaguicidas. La relación entre los plaguicidas y los síntomas musculoesqueléticos ha sido escasamente descripta en la bibliografía; sin embargo, los plaguicidas neonicotinoides ${ }^{(40)}$ tienen la misma vía de absorción que la nicotina presente en el tabaco, sugiriendo así una toxicidad similar a la de la nicotina en el cuerpo. Asimismo, algunos estudios indican que la intoxicación por plaguicidas puede estar asociada con trastornos musculoesqueléticos ${ }^{(41,42)}$, dado que los efectos neurotóxicos de los plaguicidas alteran la velocidad de conducción nerviosa ${ }^{(43)}$, que puede aumentar la percepción del dolor ${ }^{(44)}$.

\section{CONCLUSIONES}

Los estudios sobre el dolor cervical en trabajadores rurales escasean en la bibliografía. EI presente trabajo señala la importante prevalencia de dolor cervical entre agricultores que producen tabaco -en especial las mujeres- y refuerza los efectos ocupacionales asociados con la variable dependiente. El análisis estratificado nos permite observar que los factores asociados con el dolor cervical tienen especificidades de acuerdo al sexo. 
Este artículo trae a colación un tema que recibe poca atención en la bibliografía, específicamente la asociación entre los plaguicidas y la exposición a nicotina con el dolor cervical. Estos hallazgos resaltan la pertinencia de futuros estudios que permitan una mayor comprensión del papel de la exposición a químicos en los problemas musculoesqueléticos. El trabajo refuerza la importancia de cargas de trabajo ergonómicas y fisiológicas en la determinación del dolor cervical, indicando una asociación entre el uso de motosierras pesadas en ambos sexos, así como una asociación entre el enfardado de las hojas de tabaco y el trabajo en un ritmo intenso o acelerado y el dolor cervical en las mujeres.

No obstante, la evaluación de la asociación entre exposiciones ocupacionales y el dolor cervical se encuentra limitada por el bajo poder estadístico para examinar exposiciones que alcanzan un gran número de agricultores que producen tabaco -tal como el trabajo en una posición inclinada- y por el efecto del trabajador saludable, un sesgo de selección que puede estar presente en estudios transversales. Estudios futuros con muestras más grandes, estudios longitudinales, y estudios que evalúan algunas exposiciones como la altura, la masa corporal y accidentes autorreportados previos son importantes para superar las limitaciones señaladas.

Las políticas deben abordar la necesidad de mecanizar el proceso de cosecha del tabaco a fin de reducir cargas de trabajo ergonómicas y químicas que pueden tener impactos negativos en la salud. Las políticas deben promover la equidad de género, con el foco en las tareas realizadas principalmente por las mujeres, que a menudo son monótonas y repetitivas, y se superponen con el trabajo doméstico. Además, es importante promover políticas de diversificación de cultivos, con un modelo agroecológico, como forma de garantizar trabajos saludables en la agricultura familiar, sobre todo en el contexto de una reducción del consumo de tabaco a nivel mundial. Desde esta perspectiva, es esencial desarrollar políticas y proyectos para promover y monitorear la salud de las poblaciones rurales $^{(45)}$.

\section{AGRADECIMIENTOS}

Este trabajo fue posible gracias al apoyo económico del Conselho Nacional de Desenvolvimento Científico e Tecnológico (No. 483214/2009-4), y a la Fundação de Amparo à Pesquisa do Estado do Rio Grande do Sul (No. 09/0057.5).

\section{REFERENCIAS BIBLIOGRÁFICAS}

1. Anuário Brasileiro do Tabaco. Santa Cruz do Sul: Editora Gazeta Santa Cruz; 2018.

2. Pinzke S. Comparison of working conditions and prevalence of musculoskeletal symptoms among dairy farmers in Southern Sweden over a 25-year period. Front. Public Health. 2016;4:1-12. doi: 10.3389/fpubh.2016.00098.

3. Milosavljevic S, Bagheri N, Vasiljev RM, Mcbride DI, Rehn B. Does daily exposure to whole-body vibration and mechanical shock relate to the prevalence of low back and neck pain in a rural workforce? Annals of Occupational Hygiene. 2012;56(1):10-17. doi: 10.1093/annhyg/ mer068.

4. Jonsson BG, Persson J, Kilbom A. Disorders of the cervicobrachial region among female workers in the electronics industry: A two-year follow up. International Journal of
Industrial Ergonomic. 1988;3:1-12. doi: 10.1016/01698141(88)90002-9.

5. Nachemson $A L$, Jonsson $E$, (ed.). Neck and back pain: The scientific evidence of causes, diagnosis, and treatment. Philadelphia: Lippincott Williams \& Wilkins; 2000 .

6. Ohlsson K, Attewell RG, Pålsson B, Karlsson B, Balogh I, Johnsson B, Ahlm A, Skerfving S. Repetitive industrial work and neck and upper limb disorders in females. American Journal of Industrial Medicine. 1995;27(5):731-747. doi: 10.1002/ajim.4700270508.

7. Banibrata D. Gender differences in prevalence of musculoskeletal disorders among the rice farmers of West Bengal, India. Work. 2015;50(2):229-240. doi: 10.3233/ WOR-131694.

8. Riquinho DL, Hennington EA. Sistema integrado de produção do tabaco: saúde, trabalho e condições de vida de trabalhadores rurais no Sul do Brasil. Cadernos de Saúde Pública. 2016;32(12):e00072415. doi: 10.1590/0102-311X00072415.

9. Heemann F. O cultivo do fumo e condições de saúde e segurança dos trabalhadores rurais. [Dissertation of Master in Engenharia de Produção]. Porto Alegre: Universidade Federal do Rio Grande do Sul; 2009. 
10. Fassa AG, Faria NMX, Meucci RD, Fiori NS, Miranda VI, Facchini LA. Green tobacco sickness among tobacco farmers in southern Brazil. American Journal of Industrial Medicine. 2014;57:726-735. doi: 10.1002/ajim.2307.

11. Mota e Silva MS, Carvalho MGC, Moreira JC, Barreto EO, Farias KF, Nascimento CA, Silva FMN, Andrade TG, Luiz RR, Moura Neto RS, Ribeiro FL. Green tobacco sickness among Brazilian farm workers and genetic polymorphisms. BMC Research Notes. 2018;11(20):2-5. doi: 10.1186/s13104-018-3135-x.

12. Instituto Brasileiro de Geografia e Estatística. Pesquisa Nacional por Amostra de Domicilios: síntese de indicadores 2009 [Internet]. 2010 [citado 11 feb 2019]. Disponible en: https://tinyurl.com/y9dto984.

13. Barbosa Filho FH, Pessôa SA. Pessoal ocupado e jornada de trabalho: uma releitura da evolução da produtividade no Brasil. Revista Brasileira de Economia. 2014;68(2):149-169.

14. Faria NM, Rosa JA, Facchini LA. Poisoning by pesticides among family fruit farmers, Bento Gonçalves, Southern Brazil. Revista de Saúde Pública. 2009;43(2):335-344.

15. Arcury TA, Vallejos QM, Schulz MR, Feldman SR, Fleischer Jr AB, Verma A, Quandt SA. Green tobacco sickness and skin integrity among migrant Latino farmworkers. American Journal of Industrial Medicine. 2008;51(3):195-203. doi: 10.1002/ajim.20553.

16. Kuorinka I, Jonsson B, Kilbom A, Vinterberg H, BieringSørensen F, Andersson G, Jørgensen K. Standardised Nordic questionnaires for the analysis of musculoskeletal symptoms. Applied Ergonomics. 1987;18(3):233237. doi:10.1016/0003-6870(87)90010-X.

17. Pinheiro FA, Trócolli BT, Carvalho CV. Validação do Questionário Nórdico de Sintomas Osteomusculares como medida de morbidade. Revista de Saúde Pública. 2002;36(3):307-312. doi: 10.1590/S003489102002000300008 .

18. Meucci RD, Fassa AG, Paniz VM, Silva MC, Wegman $\mathrm{DH}$. Increase of chronic low back pain prevalence in a medium-sized city of southern Brazil. BMC Musculoskeletal Disorders. 2013;14:155. doi: 10.1186/1471-247414-155

19. Carvalho MP, Schmidt LG, Soares MCF. Musculoskeletal disorders and their influence on the quality of life of the dockworker: a cross-sectional study. Work. 2016;53(4):805-812. doi: 10.3233/WOR-162249.

20. Victora CG, Huttly SR, Fuchs SC, Olinto MT. The role of conceptual frameworks in epidemiological analysis: a hierarchical approach. International Journal of Epidemiology. 1997;26(1):224-227. doi:10.1093/ ije/26.1.224.

21. Jo H, Baek S, Park HW, Lee SA, Moon J, Yang JE, Kim KS, Kim JY, Kang EK. Farmers' cohort for agricultural work-related musculoskeletal disorders (FARM) study: study design, methods, and baseline characteristics of enrolled subjects. Journal of Epidemiology. 2016;26(1):50-56. doi: 10.2188/jea.JE20140271.
22. Xiao H, McCurdy SA, Stoecklin-Marois MT, Li CS, Schenker MB. Agricultural work and chronic musculoskeletal pain among latino farm workers: The MICASA study. American Journal of Industrial Medicine. 2013;56:216-225. doi: 10.1002/ajim.22118.

23. Lima RG. Desenvolvimento e relações de trabalho na fumicultura sul-brasileira. Sociologias. 2007; 9(18):190225. doi: 10.1590/S1517-45222007000200010.

24. Brandth B, Haugen MS. Rural Masculinity. In: Shucksmith M, Brown DL, (eds.). Routledge International Handbook of Rural Studies. London: Routledge; 2016. p. 412-423.

25. Cush $P$, Macken-Walsh A. Reconstituting male identities through joint farming ventures in Ireland. Sociologia Ruralis. 2018;58(4):726-744. doi: 10.1111/ soru. 12212.

26. Picavet HSJ, Schouten JSAG. Musculoskeletal pain in the Netherlands: prevalences, consequences and risk groups, the DMC3-study. Pain. 2003;102(1-2):167-178. doi:10.1016/s0304-3959(02)0372-x.

27. Leroux I, Dionne CE, Bourbonnais R, Brisson C. Prevalence of musculoskeletal pain and associated factors in the Quebec work population. International Archives of Occupational and Environmental Health. 2005;78(5):379-386. doi:10.1007/s00420-004-0578-2.

28. Fillingim RB, Edwards RR, Powell T. Sex-dependent effects of reported familial pain history on recent pain complaints and experimental pain responses. Pain. 2000;86(1-2):87-94. doi:10.1016/s03043959(00)00239-6.

29. Bernard BP. Musculoskeletal disorders and workplace factors: A critical review of epidemiological evidence for work-related musculoskeletal disorders of the neck, upper extremity, and low back pain. Cincinnati: NIOSH publication; 1997.

30. Silva A, Mattiello SM, Oliveira e Silva L, Walsh IAP. O trabalho e as LER/DORT. In: Melo MT. Trabalhador em turno: fadiga. São Paulo: Atheneu; 2013. p 41-50.

31. Tynes T, Aagestad C, Thorsen SV, Andersen LL, PerkioMakela M, Pinilla García FJ, Blanco LG, Vermeylen G, Parent-Thirion A, Hooftman W, Houtman I, Liebers F, Burr $\mathrm{H}$, Formazin M. Physical working conditions as covered in European monitoring questionnaires. BMC Public Health. 2017; 17:544. doi: 10.1186/s12889-017-4465-7

32. Mayer J, Kraus T, Ochsmann E. Longitudinal evidence for the association between work-related physical exposures and neck and/or shoulder complaints: a systematic review. International Archives of Occupational and Environmental Health. 2012;85(6):587-603. doi: 10.1007/s00420-011-0701-0.

33. Härmä M. Workhours in relation to work stress, recovery and health. Scandinavian Journal of Work, Environment \& Health. 2006;32(6):502-514. doi:10.5271/ sjweh. 1055.

34. Punnett L, Wegman DH. Work-related musculoskeletal disorders: the epidemiologic evidence and the 
debate. Journal of Electromyography and Kinesiology. 2004;14:13-23. doi: 10.1016/j.jelekin.2003.09.015.

35. Dembe $A E$, Erickson JB, Delbos RG, Banks SM. The impact of overtime and long work hours on occupational injuries and illness: new evidence from the United States. Occupational \& Environmental Medicine. 2005;62(9):588-597. doi: 10.1136/oem.2004.016667.

36. Boksem MA, Tops M. Mental fatigue: costs and benefits. Brain Research Reviews. 2008;59(1):125-139. doi: 10.1016/j.brainresrev.2008.07.001.

37. Goldenberg DL. Fibromyalgia, chronic fatigue syndrome, and myofascial pain syndrome. Current Opinion in Rheumatology. 1994;6(2):223-233.

38. Yoo SJ, Park SJ, Kim BS, Lee K, Lim HS, Kim JS, Kim IS. Airborne nicotine concentrations in the workplaces of tobacco farmers. Journal of Preventive Medicine \& Public Health. 2014;47(3):144-149. doi: 10.3961/ jpmph.2014.47.3.144.

39. Holm S, Nachemson A. A nutrition of the intervertebral disc: acute effects of cigarette smoking: An experimental animal study. Upsala Journal of Medical Sciences. 1988;93(1):91-99. doi: 10.1517/03009734000000042.

40. Tomizawa M, Casida JE. Neonicotinoid insecticide toxicology: mechanisms of selective action. Annual Review of Pharmacology and Toxicology. 2005;45:247-268. doi: 10.1146/annurev.pharmtox.45.120403.095930.
41. Meucci RD, Fassa AG, Faria NMX, Fiori NS. Chronic low back pain among tobacco farmers in southern Brazil. International Journal of Occupational and Environmental Health. 2015;21(1):66-73. doi: 10.1179/2049396714Y. 0000000094.

42. Riquinho DL, Hennington EA. Health, environment and working conditions in tabacco cultivation: a review of the literature. Ciência \& Saúde Coletiva. 2012;17(6):15871600. doi: 10.1590/S1413-8123201200060002.

43. Steenland $K$, Jenkins $B$, Ames RG, O'Malley $M$, Chrislip D, Russo J. Chronic neurological sequelae to organophosphate pesticide poisoning. American Journal of Public Health. 1994;84(5):731-736. doi: 10.2105/ AJPH.84.5.731.

44. London L, Beseler C, Bouchard MF, Bellinger DC, CoIosio C, Grandjean P, Harari R, Kootbodien T, Kromhout H, Little F, Meijster T, Moretto A, Rohlman DS, Stallones L. Neurobehavioral and neurodevelopmental effects of pesticide exposures. Neurotoxicology. 2012;33(4):887896. doi: 10.1016/j.neuro.2012.01.004.

45. Brasil, Ministério da Saúde, Secretaria de Gestão Estratégica e Participativa, Departamento de Apoio à Gestão Participativa. Política nacional de saúde integral das populações do campo e da floresta. Brasília: Editora do Ministério da Saúde; 2013.

\section{FORMA DE CITAR}

Fassa AG, Fiori NS, Meucci RD, Faria NMX, Carvalho MP. Dolor cervical entre agricultores que producen tabaco en el sur de Brasil. Salud Colectiva. 2020;16:e2307. doi: 10.18294/sc.2020.2307.

Recibido: 7 may 2019 | Versión final: 27 abr 2020 | Aprobado: 12 may 2020 | Publicado en línea: 23 jul 2020

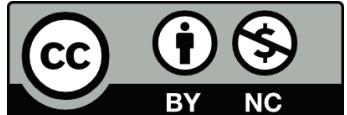

Esta obra está bajo una licencia de Creative Commons Reconocimiento-NoComercial 4.0 Internacional. Reconocimiento - Permite copiar, distribuir y comunicar públicamente la obra. A cambio, se debe reconocer y citar al autor original. No Comercial - Esta obra no puede ser utilizada con finalidades comerciales, a menos que se obtenga el permiso. 